\title{
Remote Liaison to Families: a Psychiatric Response to Medical Care Gaps Created by Pandemic Surge Conditions in New York City
}

\author{
Julie B. Penzner ${ }^{1,2} \cdot$ Jonathan D. Power ${ }^{1} \cdot$ Christopher Green $^{1} \cdot$ Joseph Mouallem ${ }^{1} \cdot$ Randi Diamond ${ }^{1}$. \\ Kimberly Hong ${ }^{1}$. Guy Maytal ${ }^{1}$
}

Received: 3 June 2020 / Accepted: 17 November 2020 / Published online: 7 January 2021

(C) Academic Psychiatry 2021

The 2019-2020 pandemic of severe acute respiratory syndrome coronavirus 2 (SARS-CoV-2) transformed life in a matter of months. Though modest respiratory virus outbreaks in the last twenty years provided test beds for responses to epidemics in Asia and the Middle East, the strain on modern medical systems caused by coronavirus disease 2019 (COVID-19) is unprecedented in living memory [1-3]. In the United States, widespread community transmission without adequate means to test, identify, and isolate the infected led to a surge of hospitalizations beginning in late winter 2020 [4]. Within weeks of its first identified case, New York City became the global epicenter of COVID-19, with over 1,000 new hospitalizations daily from late March through mid-April [5].

Caring for patients at this speed and scale required adaptation at every level of the medical system. Patient care areas were constructed in hospital atria. Treatment tents were erected in parking lots. Medical students graduated early, senior pathology residents became medical interns, ophthalmologists became emergency room doctors, and other unorthodox roles were assumed. Psychiatrists were redeployed in the manner above, and in other ways, to expand capacity.

This article describes a real-time adaptation of a psychiatry department to surge conditions: the establishment of a Remote Liaison Service for patient families, focused on supportive counseling and palliative care decisions, and staffed by psychiatric residents. Developed jointly by the Departments of Psychiatry and Palliative Medicine at Weill Cornell Medicine, this service benefitted families, lessened demands on on-site providers, and enhanced patient care. All services agreed it would be re-implemented in future surges. It was a

Julie B. Penzner

julie.penzner@duke.edu

1 Weill Cornell Medicine, New York, NY, USA

2 Duke University, Durham, NC, USA valuable educational experience. We write to outline this Remote Liaison Service, which should be of interest to all clinicians experiencing surge conditions.

\section{Rationale for the Remote Liaison Service}

The Remote Liaison Service was developed to address a pandemic-specific gap in care, namely, a severe decrease in the quality of communication between providers and the families making important decisions for incapacitated patients. During the surge, visitors were restricted entirely or only allowed when death was imminent. A "typical" family of a patient in the intensive care unit (ICU) in early April 2020 likely had not seen the patient since they called 911 weeks prior, were probably interacting with their third or even fifth medical team (depending on transfers between and within institutions), and received a daily few-minute telephone update. Patient clinical statuses often declined abruptly before communication of wishes, and ventilation courses were long. Provider ability to facilitate video chat was limited by clinical demands, patient sedation, and loud machinery. Family stress was compounded by uncertainty about the disease, personal stresses of quarantine or illness, and social and economic upheaval. It became clear that a more robust service was needed to support the families of COVID-19 patients.

The Remote Liaison Service was developed specifically to assist medical providers working in novel and stressful situations, including in teams with missing members due to quarantine or hospitalization with COVID-19, and in teams with new members outside of their usual setting and skill. Teams were tasked with treating a novel illness with high acuity and a tendency for sudden decompensation, that was also a highly transmissible infectious disease requiring personal protective equipment. Patients were unusually young, often formerly healthy and now gravely ill, who may not have had conversations about death with their families. Illness guidelines and 
clinical trials changed frequently, and staff-to-patient ratio was lower than usual. The medical team therefore had limited time for family communication.

\section{Elements and Scope of the Remote Liaison Service}

To address these needs, and in conjunction with the Palliative Care Service, the Psychiatry Department created a new remote liaison role for psychiatric residents. The Remote Liaison Service would support the families of patients with COVID-19 while easing the communication responsibilities of the treating team. Table 1 outlines the elements and scope of this service.

Utilization of the Remote Liaison Service began by on-site medical team or social worker request. An on-site "point person" from palliative care distributed cases to fourth-year psychiatry residents. Each resident was assigned several cases per week while continuing their normal outpatient telehealth duties. Upon receiving the case, the resident psychiatrist would call the family, explain their role and, with the family's consent, follow up two or three times weekly or as needed by phone. The psychiatry residents decided the level of engagement with families. Call length depended on the family's wishes. Interpreters were often used. Calls were documented in the patient chart. Remote liaisons interacted with the primary teams through the patient chart rather than directly, in order to avoid encumbering the primary teams. Support calls

\section{Table 1 Elements of the Remote Liaison Service}

Initiation of service
Medical teams, palliative care, and social workers could request
Palliative care "point person" distributed cases and monitored charts
Psychiatry residents accepted cases while continuing normal duties
Palliative care "point person" invoked formal palliative care as needed
Service structure
Tracked patients in medical chart; uninvolved in direct care
Called families to introduce themselves and the service
Followed up 2-3 times per week or as needed
Documented call content in medical chart
Calls lasted $~ 5-45$ min depending on needs and issues
Depth of engagement flexible
Utilized phone interpreter as needed
Cases supervised weekly by psychiatrists
Termination of service
Upon family request, patient discharge, or patient death
Palliative service devised after-care group program for families
Potential alterations for next generation of the service
Many kinds of providers could staff, beyond psychiatry residents
Weekly rounds with full palliative care team
To efficiently flag new/impending/potential issues
Potentially blended with psychiatric supervision
Have clear policy for when calls would end in cases of discharge or
death
Have pre-developed after-care plan for families
Cases become continuity cases and do not rotate between rotating staff

continued until families felt they were unnecessary, the patient recovered, or the patient died. Of note, the Palliative Care Service developed a family group program that assumed post-hospitalization care for interested families. Weekly remote supervision for the psychiatry residents was provided by attending psychiatrists who specialized in palliative care and consultation-liaison psychiatry. A group format was selected for supervision for efficiency, and to enable residents to learn from one another's work.

The scope of the calls included patient-centric and familycentric aspects. The psychiatry residents assessed what the family understood of the medical situation and the quality of discussions with the medical teams. Psychiatry residents discussed possible outcomes and views on death but also made it clear to families that decisions about Advanced Directives or specific interventions needed to be discussed with the primary or palliative care teams. The Palliative Care Team conducted needed goals-of-care discussions. The families themselves, though not formally "patients", were also a target of service. Psychiatry residents provided supportive counseling to the families, normalizing their grief and anxiety, listening to their fears and hopes, and learning about the patient's personality. The psychiatric residents assessed families for problematic stress reactions and tried to guide them toward healthy coping mechanisms, often linking families to general services (e.g., food, unemployment) or specific mental health services.

This service was implemented in early April 2020, just as cases crested in New York City. Ultimately, 25 families were referred during the surge. Ages of patients ranged from 32 82 years old with average age of 62 . Engagement with families lasted 1-42 days with an average of 13 days, and calls were documented 1-11 times per family, averaging 4 notes, coming about once every $2-3$ days. About $30 \%$ of cases ended with family feeling it was no longer needed, the remainder ended at death or discharge.

\section{Benefits and Lessons Learned}

The remote liaison program conferred a number of benefits. Families gained a constant point of contact despite changes in treatment teams and settings. Remote liaison psychiatrists had adequate and flexible time to conduct discussions in order to ensure that the "big picture" was grasped by families with varying medical literacy. This facilitated the daily updates with the medical team and addressed treatment goals. Onsite clinicians diverted work to off-site providers who were eager to help. Psychiatrists provided care to people who were themselves quarantined and under incredible stress, but who generally could not access psychiatric care during the surge. The sustained contact with families allowed residents to lead urgent discussions about the possibility and meaning of death, 
a role that is rarely encountered in training. By remaining separate from the treating team, the remote liaison could absorb frustrations of the families, and could work through these issues to help family alignment with treatment teams. Many families expressed gratitude for the calls and the connection they provided, even in negative outcomes. This response was probably amplified by the attenuated contact families had with patients and the treating team during surge conditions.

The palliative care team appreciated the Remote Liaison Service and will implement it again in a future surge. Logistical issues were smoothed by assigning a "point person" on palliative care to interface with the remote liaison staff. In the next iteration, we would implement once-weekly rounds between the palliative care team and the remote liaison staff to outline priorities, troubleshoot issues, and strengthen crossdisciplinary alliance.

This particular service was staffed by senior psychiatry residents, and many of them stated that they would volunteer for such a service again. Many, especially those not working on-site during the surge, found it deeply meaningful. All believed that families were aided by the service, especially by continuity of contact. As previously indicated, this service was implemented at a relatively small scale, but it proved useful.

\section{Illustrative Cases Discussion}

We close with two cases illustrating the role of the service, with alterations to mask identity.

Case 1: A 68-year-old male with diabetes and a seizure disorder presented to an outside hospital with altered mental status after a fall and was found to be COVID-19 positive. He required intubation on hospital day 3 , and transferred to our hospital a week later, both without his family's knowledge. The patient was full code and remained intubated and sedated in the ICU for 15 days with renal failure, hemodialysis, and eventual tapering of sedation but persistent altered mental status without seizures. Remote liaison service was requested by the medical team during his ICU stay. The family had been angry that they were not notified about his intubation and transfer, and had had gained a reputation as "difficult". By assuming an outside-of-treating-team role, the remote liaison created a safe space for the family to express disappointment. The liaison normalized their response and educated them on the known courses of COVID-19 patients, helping them understand why and how medical decisions were made. Other topics of discussion included coping mechanisms for overwhelming sadness. After several calls, there was a strong bond to the psychiatrist, which facilitated discussions of the likelihood of death given prolonged illness and minimal progress. The family either had not had these discussions, or was not "hearing" the medical team, and was able to work through the patient's and family's wishes with the remote liaison psychiatrist. They expressed relief that someone was "finally talking about it". Certainly, it was easier to broach this topic via repeated conversations with sufficient time available, an impossible task for the on-site medical team.

Case 2: A 72-year-old female with an unspecified arrhythmia presented to her local hospital in respiratory failure with COVID-19. She was intubated in the emergency room and transferred to our hospital within days of admission due to acute kidney injury and atrial fibrillation. On day 9, hemoglobin dropped several points in the setting of increased pressor support. A large hematoma was noted at the site of a previous arterial line, leading to discovery of a large pseudoaneurysm. The patient became severely hypotensive, was made do not resuscitate by her daughter, and deteriorated over several days. The daughter visited her mother on the day of death at the ICU team's invitation, and the ICU team requested a remote family liaison after witnessing the daughter's distress. The psychiatrist spoke to the daughter that afternoon for over an hour. She was wracked with grief and guilt. She had a limited understanding of the situation, believing that her mother "lost blood" and that she could have given blood during the visit to the ICU to save her mother. The psychiatrist provided supportive counseling, validated the daughter's grief, gently explained the medical situation in terms she could understand, and ensured that she had family on hand to support her at her home. A call several days later was also made to connect her with therapists who spoke her native language.

These cases illustrate important, stressful, timesensitive situations for families. During a pandemic, especially during a surge when resources of all kinds are stretched or exhausted, there is limited support set aside for patient families. The kinds of stresses that patient families will experience are familiar to disaster psychiatry, and are anticipated and addressed in approaches like Psychological First Aid [6]. But the slow-motion unfolding and persistent social distancing inherent to pandemics causes a particular kind of isolation for families, affecting relationships within the family, relationships to the patient, and relationships with medical providers [7]. Psychiatrists are well-suited to understand the emotional and physical reactions to these situations and are wellpositioned to provide a helpful bridge between medical teams and families. Modern medical systems have not undergone stress tests like pandemic surges until the present moment. Our Remote Liaison Service was an attempt to address, in real time, a care gap that emerged. The service decreased burdens on providers of direct patient care, aided families, and enriched the experience and practice of the involved psychiatric residents. The burden on remote liaison staff already working remotely was relatively light, and we suggest that such a service may be both feasible and beneficial for patient care during future pandemic surges. 
Acknowledgments We thank everyone that helped patients and their families during the 2020 COVID-19 crisis. We thank Rachel Jaffe, NP for her teaching about palliative care.

\section{Compliance with Ethical Standards}

Disclosures The authors declare no conflicts of interest. Protected Health Information is not included in this manuscript, and the work is compliant with the Health Insurance Portability and Accountability Act.

\section{References}

1. Singer PA, Benatar SR, Bernstein M, et al. Ethics and SARS: lessons from Toronto. BMJ. 2003;327(7427):1342-4.
2. Wadhera RK, Wadhera P, Gaba P, et al. in COVID-19 hospitalizations and deaths across New York City boroughs. JAMA. 2020;323(21):2192-5.

3. Atkinson C. G20 leaders must answer to COVID-19. Science. 2020;368(6487):111.

4. Richardson S, Hirsch JS, Narasimhan M, et al. Presenting characteristics, comorbidities, and outcomes among 5700 patients hospitalized with COVID-19 in the New York City area. JAMA. 2020;323(20):2052-9.

5. Goyal P, Choi JJ, Pinheiro LC, et al. Clinical Characteristics of Covid-19 in New York City. N Engl J Med. 2020;382(24):2372-4.

6. Pekevski J. First responders and psychological first aid. J Emerg Manag. 2013;11(1):39-48.

7. Huremovic D. Psychiatry of pandemics: a mental health response to infection outbreak. Cham: Springer; 2019. 